\title{
Could Endorphins Participate in the Limbic Pathways Responsible for NDEs After Acute Cerebral Hypoxia?
}

To the Editor:

Near-death experiences (NDEs) have been provoked by T. Lempert, M. Bauer, and D. Schmidt (1995) in healthy volunteers by experimentally induced syncope, through hyperventilation and Valsalva maneuver. Those authors concluded that acute cerebral hypoxia may induce an agonal limbic syndrome responsible for visual and auditory hallucinations, most of them pleasant and peaceful, whose characteristics were rather similar to those described in reports of NDEs. We have measured brain opioids in cerebrospinal fluid and brain tissue of dogs subjected to acute cerebral ischemia and hypoxia secondary to cardiac arrest, and found a significant increase of B-endorphin immediately after the induction of hypoxia (Sotelo, Perez, Guevara, and Fernandez, 1995). Our findings could explain the "pleasant, detached and peaceful" experiences reported by the subjects with experimental syncope through a sudden liberation of brain opioids induced by acute cerebral hypoxia.

The comparison of NDEs with experiences due to recreational drugs (Siegel, 1978), particularly opiates (Lipp, 1991), supports the idea that endogenous opioids could participate in the limbic syndrome 
of NDEs. The fact that these experiences are particularly frequent in survivors of cardiac arrest (Sabom and Kreutziger, 1977) may indicate that sudden hypoxia in a normal functioning brain activates limbic-mediated pathways related with complex pleasant and peaceful mental perceptions, in which endogenous opioids could play a crucial role. Moreover, a limbic syndrome could be an ancestral brain mechanism of analgesia and sedation developed to mitigate the natural pain and fear associated with the conscious perception of the proximity of death. If this is true, then NDEs should be more conspicuous in subjects with sudden brain hypoxia than in subjects with structural or metabolic brain damage.

\section{References}

Lempert, T., Bauer, M., and Schmidt, D. (1994). Syncope and near-death experience. Lancet, 344, 829-830.

Lipp, J. (1991). Possible mechanisms of morphine analgesia. Clinical Neuropharmacology, 14, 131-147.

Sabom, M. B., and Kreutziger, S. (1977). Near-death experiences [Letter]. New England Journal of Medicine, 297, 1071.

Siegel, R. K (1978). Phencyclidine and ketamine intoxication: A study of recreational users. In R. C. Peterson and R. C. Stillman (Eds), Phencyclidine abuse: An appraisal (pp. 119-150). Rockville, MD: National Institute of Drug Abuse.

Sotelo, J., Perez, R., Guevara, P., and Fernandez, A. (1995). Changes in brain, plasma and cerebrospinal fluid contents of Bendorphin in dogs at the moment of death. Neurological Research, 17, 223-225.

Patricia Guevara, M.Sc.

Julio Sotelo, M.D.

Instituto Nacional de Neurologia y Neurocirugia

Insurgentes Sur 3877

14269 Mexico City

Mexico 